\title{
Pole Placement with Fields of Positive Characteristic
}

\author{
Elisa Gorla and Joachim Rosenthal
}

\begin{abstract}
The pole placement problem belongs to the classical problems of linear systems theory. It is often assumed that the ground field is the real numbers $\mathbb{R}$ or the complex numbers $\mathbb{C}$.

The major result over the complex numbers derived in 1981 by Brockett and Byrnes states that arbitrary static pole placement is possible for a generic set of $m$-inputs, $p$-outputs and McMillan degree $n$ system as soon as $m p \geq n$. Moreover the number of solutions in the situation $m p=n$ is an intersection number first computed by Hermann Schubert in the 19th century.

In this paper we show that the same result with slightly different proofs holds over any algebraically closed field.
\end{abstract}

\section{Introduction}

Let $\mathbb{F}$ be an arbitrary field and let $A, B, C$ be matrices of size $n \times n, n \times m$ and $p \times n$, with entries in $\mathbb{F}$. These matrices define a discrete time dynamical system through the equations:

$$
\begin{aligned}
x(t+1) & =A x(t)+B u(t) \\
y(t) & =C x(t) .
\end{aligned}
$$

An $m \times p$ matrix $K$ with entries in $\mathbb{F}$ defines the feedback law:

$$
u(t)=K y(t) .
$$

Elisa Gorla

Department of Mathematics, University of Basel, Basel, Switzerland http://www.math.unibas.ch

Joachim Rosenthal

Institute of Mathematics, University of Zürich, Zürich, Switzerland

http://www.math.uzh.ch/aa 
Applying (2) to the system (11), one gets the closed loop system:

$$
x(t+1)=(A+B K C) x(t) .
$$

The static output pole placement problem asks for conditions on the matrices $A, B, C$ which guarantee that the characteristic polynomial of the closed loop system, i.e., the characteristic polynomial of the matrix $(A+B K C)$ can be made arbitrary.

We can explain this problem also in terms of the so-called pole placement map. For this, identify the set of monic polynomials of degree $n$ of the form:

$$
s^{n}+a_{n-1} s^{n-1}+\cdots+a_{1} s+a_{0} \in \mathbb{F}[s]
$$

with the vector space $\mathbb{F}^{n}$. Then we are seeking conditions which guarantee that the pole placement map:

$$
\chi_{(A, B, C)}: \mathbb{F}^{m \times p} \longrightarrow \mathbb{F}^{n}, \quad K \longmapsto \operatorname{det}(s I-A-B K C)
$$

is surjective, or at least that the image contains a non-empty Zariski-open set.

Many facets of this problem have been studied in the literature and the reader is referred to [2, 10, 14, 15] where also more references to the literature can be found.

If the base field is the complex numbers, then the major result is due to Brockett and Byrnes [1]:

Theorem 1. If the base field $\mathbb{F}$ equals $\mathbb{C}$, the complex numbers, then $\chi$ is surjective for generic matrices $A, B, C$ if and only if $m p \geq n$. Moreover if $m p=n$ and $\chi$ is surjective, then the general fiber $\chi^{-1}(\phi)$ has cardinality

$$
d(m, p)=\frac{1 ! 2 ! \cdots(p-1) !(m p) !}{m !(m+1) ! \cdots(m+p-1) !} .
$$

In the next section we will go over the proof of Theorem 1 in the situation when the base field $\mathbb{F}$ is algebraically closed and has characteristic zero. In Section 3 we will address the difficulties which occur in positive characteristic. The main result of the paper is a proof that Theorem 1 holds over any algebraically closed field in the case $n=m p$.

\section{Connection to Geometry and a proof of Theorem 1in characteristic zero}

Consider the transfer function $G(s):=C(s I-A)^{-1} B$ and a left coprime factorization:

$$
G(s)=D^{-1}(s) N(s)=C(s I-A)^{-1} B .
$$

Over any field $\mathbb{F}$ we have the property that the $p \times(m+p)$ matrix $[N(s) D(s)]$ has rank $p$ when evaluated at an arbitrary element of the algebraic closure $\overline{\mathbb{F}}$ of $\mathbb{F}$. In 
other words if $\lambda \in \overline{\mathbb{F}}$ then

$$
\operatorname{rank}[N(\lambda) D(\lambda)]=p
$$

It was the insight of Hermann and Martin [5] to realize that every linear system $G(s)$ naturally defines a rational map into the Grassmann variety $\operatorname{Grass}\left(p, \mathbb{F}^{m+p}\right)$ :

$$
h: \mathbb{P}^{1} \longrightarrow \operatorname{Grass}\left(p, \mathbb{F}^{m+p}\right), s \longmapsto \operatorname{rowsp}[N(s) D(s)] .
$$

The map $h$ does not depend on the coprime factorization, and two different linear systems $G_{1}(s)$ and $G_{2}(s)$ have different associated rational maps. By the previous remark, the map is well defined for every element $\lambda \in \overline{\mathbb{F}}$. For this reason one usually refers to $h$ as the Hermann-Martin map associated to the linear system $G(s)$.

In order to arrive at an algebraic geometric formulation of the pole placement problem, consider a left coprime factorization $G(s)=D^{-1}(s) N(s)$ with the property that $\operatorname{det}(s I-A)=\operatorname{det} D(s)$. Then it is well known that the closed loop characteristic polynomial can also be written as:

$$
\operatorname{det}(s I-A-B K C)=\operatorname{det}\left[\begin{array}{cc}
I & K \\
N(s) & D(s)
\end{array}\right] .
$$

Assume now that a desired closed loop characteristic polynomial $\phi(s)$ factors over the algebraic closure as:

$$
\phi(s)=\prod_{i=1}^{n}\left(s-s_{i}\right), s_{i} \in \overline{\mathbb{F}}, i=1, \ldots, n .
$$

The condition $\operatorname{det}(s I-A-B K C)=\phi(s)$ then translates into the geometric condition:

$$
\operatorname{rowsp}[I K] \bigcap \operatorname{rowsp}\left[N\left(s_{i}\right) D\left(s_{i}\right)\right] \neq\{0\}, i=1, \ldots, n .
$$

This formulation is closely connected to a theorem due to Hermann Schubert:

Theorem 2. Given $n$-dimensional subspaces $U_{i} \subset \mathbb{C}^{m+p}$. If $n \leq m p$, then there is an m-dimensional subspace $V \subset \mathbb{C}^{m+p}$ such that

$$
V \bigcap U_{i} \neq\{0\}, i=1, \ldots, n .
$$

Moreover if $n=m p$ and the subspaces $U_{i}$ are in "general position", then there are exactly $d(m, p)$ (see Equation (5)) different solutions $V \subset \mathbb{C}^{m+p}$ satisfying Condition (77).

Theorem 2 was derived by Hermann Schubert towards the end of the 19th century [11, 12]. The mathematicians at the time were not convinced with the proofs Schubert was providing. The verification of the statements constituted Hilbert's 15 th problem, which he presented at the International Congress of Mathematics in 1900 in Paris. Theorem 2 has been later verified rigorously and we refer to Kleiman's survey article [4]. 
It is not completely obvious how the geometric result of Schubert implies Theorem 1 of Brockett and Byrnes. The following questions have to be addressed:

1. Given an $m$-dimensional subspace rowsp $\left[K_{1} K_{2}\right] \subset \mathbb{C}^{m+p}$, where $K_{1}$ is an $m \times m$ matrix and $K_{2}$ is an $m \times p$ matrix. Assume rowsp $\left[K_{1} K_{2}\right] \subset \mathbb{C}^{m+p}$ is a geometric solution, i.e.,

$$
\operatorname{det}\left[\begin{array}{cc}
K_{1} & K_{2} \\
N\left(s_{i}\right) & D\left(s_{i}\right)
\end{array}\right]=0, i=1, \ldots, n .
$$

Does it follow that $\left[K_{1} K_{2}\right]$ is row equivalent to $[I K]$ and $K$ represents a feedback law? For this to happen it is necessary and sufficient that $K_{1}$ is invertible.

2. Assume rowsp $\left[K_{1} K_{2}\right] \subset \mathbb{C}^{m+p}$ is a geometric solution in the sense of (8). Does it follow that $\operatorname{det}\left[\begin{array}{cc}I & K \\ N(s) & D(s)\end{array}\right]$ is NOT the zero polynomial?

3. How is it possible to deal with multiple roots?

These questions were all addressed in [1]. A key ingredient is the notion of nondegenerate system.

Definition 1. An $m$-input, $p$-output linear system $G(s)=D^{-1}(s) N(s)$ is called degenerate, if there exist an $m \times m$ matrix $K_{1}$ and an $m \times p$ matrix $K_{2}$ such that $\left[K_{1} K_{2}\right]$ has full rank $m$ and

$$
\operatorname{det}\left[\begin{array}{cc}
K_{1} & K_{2} \\
N(s) & D(s)
\end{array}\right]=0 .
$$

A system $G(s)$ which is not degenerate will be called non-degenerate.

In more geometric terms, the Hermann Martin curve associated to a non-degenerate system does not lie in any Schubert hyper-surface.

If $[N(s) D(s)]$ represents a non-degenerate system of McMillan degree $n$, then

$$
\operatorname{det}\left[\begin{array}{cc}
K_{1} & K_{2} \\
N(s) & D(s)
\end{array}\right] \neq 0
$$

for any $\left[K_{1} K_{2}\right]$ of full rank. If in addition $\left[K_{1} K_{2}\right]$ is a geometric solution, then Condition (8) is satisfied and det $\left[\begin{array}{cc}K_{1} & K_{2} \\ N(s) & D(s)\end{array}\right]$ is a polynomial of degree at least $n$. All the full size minors of $[N(s) D(s)]$ have degree less than $n-1$, with the exception of the determinant of $D(s)$, which has degree $n$. So the polynomial det $\left[\begin{array}{cc}K_{1} & K_{2} \\ N(s) & D(s)\end{array}\right]$ cannot have degree $n$ unless $K_{1}$ is invertible. Hence it follows that a geometric solution for a non-degenerate system results in a feedback solution $u=K y$ on the systems theory side.

Non-degenerate systems are therefore very desirable. The following theorem was formulated in [1] in the case when the base field is the complex numbers.

Theorem 3. Let $\mathbb{F}$ be an arbitrary field. If $n<m p$ then every system $(A, B, C)$ defined over $\mathbb{F}$ with m-inputs, p-outputs and McMillan degree $n$ is degenerate. If $\mathbb{F}$ is an algebraically closed field and $n \geq m p$, then a generic system $(A, B, C) \in \mathbb{F}^{n^{2}+n(m+p)}$ is non-degenerate. 
The proof of the first part of the statement follows from basic properties of coprime factorizations of transfer functions. Indeed let the $p \times(m+p)$ polynomial matrix $M(s)=[N(s) D(s)]$ represent an $m$-inputs, $p$-outputs system of McMillan degree $n<m p$. Then, possibly after some row reductions, we find a row of $M(s)$ whose degree is at most $m-1$. Using this row one readily constructs a full rank $m \times(m+p)$ matrix such that (9) holds. This shows that $G(s)=D^{-1}(s) N(s)$ is degenerate.

The second part of the statement, namely that a generic system defined over $\mathbb{F}$ is non-degenerate, will be established through a series of lemmas. Here $\mathbb{F}$ is an algebraically closed field of characteristic zero.

Notice that it is enough to show that the set of degenerate systems is contained in a proper algebraic set of $\mathbb{F}^{n^{2}+n(m+p)}$. In order to prove this, we establish an algebraic relation between the polynomial matrix $[N(s) D(s)]$ and the matrices $(A, B, C)$. The following lemma is an ingredient of classical realization theory. The proof and the concept of basis matrix is found in [9].

Lemma 1. Assume $G(s)=D^{-1}(s) N(s)$ is a left coprime factorization of a $p \times m$ transfer function of McMillan degree $n$. Then for every $p \times n$ basis matrix $X(s)$ there are matrices $A \in \mathbb{F}^{n \times n}, B \in \mathbb{F}^{n \times m}$ and $C \in \mathbb{F}^{p \times n}$ such that:

$$
\operatorname{ker}_{\mathbb{F}(s)}[X(s)|N(s)| D(s)]=\operatorname{im}_{\mathbb{F}(s)}\left[\begin{array}{cc}
\left(s I_{n}-A\right) & B \\
0 & I_{m} \\
C & 0
\end{array}\right] .
$$

Furthermore $(A, B, C)$ is a minimal realization of $G(s)$, i.e.,

$$
G(s)=C(s I-A)^{-1} B,
$$

and for every minimal realization $(A, B, C)$ of $G(s)$ there exists a basis matrix $X(s)$ such that (10) is satisfied.

As pointed out in [9], for certain basis matrices $X(s)$ it is possible to compute $(A, B, C)$ just "by inspection".

Using the previous lemma, one readily establishes the following:

Lemma 2. Assume that $(A, B, C)$ is a minimal realization of $G(s)=D^{-1}(s) N(s)$ and $\operatorname{det}(s I-A)=\operatorname{det} D(s)$. Then

$$
\operatorname{det}\left[\begin{array}{cc}
K_{1} & K_{2} \\
N(s) & D(s)
\end{array}\right]=\operatorname{det}\left[\begin{array}{cc}
s I-A & B \\
K_{2} C & K_{1}
\end{array}\right] .
$$

As before, identify an $m$-inputs, $p$-outputs system $(A, B, C)$ of McMillan degree $n$ with a point of $\mathbb{F}^{n^{2}+n(m+p)}$. Let $S$ be the set:

$$
\left\{\left(\left(K_{1}, K_{2}\right),(A, B, C)\right) \in \operatorname{Grass}\left(m, \mathbb{F}^{m+p}\right) \times \mathbb{F}^{n^{2}+n(m+p)}: \operatorname{det}\left[\begin{array}{cc}
s I-A & B \\
K_{2} C & K_{1}
\end{array}\right]=0\right\} .
$$


Since $\operatorname{Grass}\left(m, \mathbb{F}^{m+p}\right)$ is a projective variety, the projection of $S$ onto $\mathbb{F}^{n^{2}+n(m+p)}$ is an algebraic set. This follows from the main theorem of elimination theory (see, e.g., [6]). We have therefore established that the set of degenerate systems inside $\mathbb{F}^{n^{2}+n(m+p)}$ is an algebraic set. We establish the genericity result as soon as we can show the existence of one non-degenerate system, under the assumption that $n \geq m p$.

Remark 1. In the case of proper transfer functions, the dimension of the coincidence set $S$ was computed in [8, Theorem 5.5]. With this result it was then shown in [8] that the set of non-degenerate systems inside the quasi-projective variety of proper transfer functions contains a dense Zariski-open set as soon as $n \geq m p$.

Definition 2. Let $\mathbb{F}$ be an algebraically closed field of characteristic 0 . The osculating normal curve $C_{p, m}$ is the closure of the image of the morphism

$$
\begin{aligned}
\mathbb{F} & \longrightarrow \operatorname{Grass}\left(p, \mathbb{F}^{m+p}\right) \\
s & \longmapsto \operatorname{rowsp}\left[\frac{d}{d^{s}} j^{j}\right]_{i=0, \ldots, p-1 ; j=0, \ldots, m+p-1}
\end{aligned}
$$

We denote by $d / d^{i}$ the $i$-th derivative with respect to $s$, i.e.,

$$
\frac{d}{d^{i}} s^{j}= \begin{cases}\prod_{k=0}^{i-1}(j-k) s^{j-i} & \text { if } j \geq i \\ 0 & \text { if } j<i .\end{cases}
$$

The osculating normal curve is an example of a non-degenerate curve in the Grassmannian $\operatorname{Grass}\left(p, \mathbb{F}^{m+p}\right)$. An elementary matrix proof of this fact was first given in [7]. We will say more about it in the next section. If $n>m p$ one constructs a non-degenerate system by simply multiplying the last column of the matrix representing the osculating normal curve by $s^{n-m p}$.

In the case $p=1$, this is the rational normal curve of degree $m$ in $\mathbb{P}^{m} \cong$ $\operatorname{Grass}\left(1, \mathbb{F}^{m+1}\right)$. In the case $m=1$, the osculating normal curve is isomorphic to the rational normal curve of degree $p$ in $\mathbb{P}^{p} \cong \operatorname{Grass}\left(p, \mathbb{F}^{p+1}\right)$.

So far we have shown that if $m p \geq n$, then a generic system is non-degenerate. Moreover, if $n=m p$, the system is non-degenerate and the desired closed loop polynomial has distinct roots, then pole placement is possible with $d(m, p)$ different feedback compensators.

It remains to be addressed the question of multiple roots in the closed loop polynomial. This has been done in the literature by lifting the pole placement map (4) from $\mathbb{F}^{m \times p}$ to the Grassmann variety $\operatorname{Grass}\left(m, \mathbb{F}^{m+p}\right)$. We follow the arguments in [10].

We can expand the closed loop characteristic polynomial as:

$$
\operatorname{det}\left[\begin{array}{cc}
K_{1} & K_{2} \\
N(s) & D(s)
\end{array}\right]=\sum_{\alpha} k_{\alpha} g_{\alpha}(s)
$$

where $k_{\alpha}$ are the Plücker coordinates of rowsp $\left[K_{1} K_{2}\right] \in \operatorname{Grass}\left(m, \mathbb{F}^{m+p}\right)$ and where the polynomials $g_{\alpha}(s)$ are (up to sign) the corresponding Plücker coordinates of $[N(s) D(s)]$. Let $\mathbb{P}^{N}$ be the projective space $\mathbb{P}\left(\wedge^{m} \mathbb{F}^{m+p}\right)$ and let 


$$
E_{(A, B, C)}:=\left\{k \in \mathbb{P}^{N} \mid \sum_{\alpha} g_{\alpha}(s) k_{\alpha}=0\right\} .
$$

As shown in [15], one has an extended pole placement map with the structure of a central projection:

$$
L_{(A, B, C)}: \mathbb{P}^{N}-E_{(A, B, C)} \longrightarrow \mathbb{P}^{n}, \quad k \longmapsto \sum_{\alpha} k_{\alpha} g_{\alpha}(s) .
$$

A system $[N(s) D(s)]$ is non-degenerate if and only if:

$$
E_{(A, B, C)} \cap \operatorname{Grass}\left(m, \mathbb{F}^{m+p}\right)=\{\} .
$$

For a non-degenerate system, the extended pole placement map $L_{(A, B, C)}$ induces a finite morphism:

$$
\hat{\chi}_{(A, B, C)}: \operatorname{Grass}\left(m, \mathbb{F}^{m+p}\right) \longrightarrow \mathbb{P}^{n}, \quad \operatorname{rowsp}\left[K_{1} K_{2}\right] \longmapsto \operatorname{det}\left[\begin{array}{cc}
K_{1} & K_{2} \\
N(s) & D(s)
\end{array}\right] .
$$

The inverse image of a closed loop polynomial $\phi(s) \in \mathbb{P}^{n}$ under the map $L_{(A, B, C)}$ is a linear space which intersects the Grassmann variety $\operatorname{Grass}\left(m, \mathbb{F}^{m+p}\right)$ in as many points (counted with multiplicity) as the degree of the Grassmann variety. This is equal to Schubert's number $d(m, p)$.

This completes the proof of Theorem 1 of Brockett and Byrnes in the case $n=m p$ not only for the field of complex numbers, but also in the case when the base field is algebraically closed and has characteristic zero. In Remark 8 we will discuss how to extend the proof to the case when $n<m p$.

In the next section we will discuss how to extend Theorem 3 to the case of an algebraically closed field of positive characteristic. We will show that it is much more tricky to establish the existence of non-degenerate systems in the case when the base field has positive characteristic.

\section{A proof of Theorem 1 1 in positive characteristic}

Let $\mathbb{F}$ be an algebraically closed field of characteristic $q>0$. Lemma 1 and Lemma 2 as formulated in the last section only depend on techniques from linear algebra and are true over an arbitrary field, so in particular over an algebraically closed field.

If $X$ is a projective variety, $Y$ is a quasi-projective variety, and $S \subset X \times Y$ is an algebraic subset, then the projection of $S$ onto $Y$ is a Zariski-closed subset of $Y$ (see, e.g., [13, Chapter I, Section 5.2]). This shows that Theorem 3 also holds over an algebraically closed field. In order to establish Theorem 1 we have to show that there exists at least one non-degenerate system for any choice of the parameters $p, m, n \geq m p$. We will also show that a generic fiber contains $d(m, p)$ elements when $n=m p$. 
The last statement is true as soon as the extended pole placement map $\hat{\chi}_{(A, B, C)}$ is separable [13, Chapter II, Section 6.3]. This is indeed the case: $\hat{\chi}_{(A, B, C)}$ can be seen as the composition of the Plücker embedding (which just involves the computation of minors) and the linear map $L_{(A, B, C)}$. Both maps are separable, and we conclude therefore that the composition map is separable.

So there remains the problem of establishing the existence of non-degenerate systems in the case $n \geq m p$. As we will show next, the osculating normal curve may be degenerate in characteristic $q>0$. In Section 3.2 we will provide alternative examples of non-degenerate systems in positive characteristic, while in Section 3.3 we will discuss the case of finite fields.

\subsection{The osculating normal curve}

Although the osculating normal curve is defined over a field of characteristic zero, its reduction modulo $q$ defines a curve in $\operatorname{Grass}\left(p, \mathbb{F}^{m+p}\right)$, which can again be regarded as the closure of the image of morphism (13). If $p=1$, the curve is the rational normal curve of degree $m$ in $\operatorname{Grass}\left(1, \mathbb{F}^{m+1}\right) \cong \mathbb{P}^{m}$. In particular it is nondegenerate. Notice however that the reduction of the osculating normal curve is degenerate whenever $q \leq p+m$, provided that $p \geq 2$. This is easily checked if $q<p$, since in this case the $(q+1)$-st row of the matrix defining the curve is identically zero. If $p \leq q \leq p+m$, consider the minor of the sub-matrix consisting of columns $1, \ldots, p-1, q$. This sub-matrix has the form:

$$
\left[\begin{array}{ccccc}
1 & s & \ldots & s^{p-2} & s^{q} \\
0 & 1 & & (p-2) s^{p-3} & 0 \\
\vdots & & \ddots & \vdots & \vdots \\
\vdots & & (p-2) ! & \vdots \\
0 & \ldots & & 0 & 0
\end{array}\right]
$$

It follows that the corresponding minor is zero. By choosing a compensator $\left[K_{1} K_{2}\right]$ whose sub-matrix consisting of the "complementary columns" $p, p+1, \ldots, q-$ $1, q+1, \ldots, p+m$ is the identity matrix and where all other elements are zero, one verifies that the osculating normal curve is also degenerate in this situation.

Remark 2. If at least one minor of the matrix $[N(s) D(s)]$ is 0 , then the system $G(s)=D^{-1}(s) N(s)$ is degenerate.

Notice that if $q \gg 0$, then the reduction modulo $q$ of the osculating normal curve is non-degenerate. This reflects the usual fact that "fields with large enough characteristic behave like fields of characteristic zero".

The appearance of many zero entries in the matrix over a field $\mathbb{F}$ of "small" positive characteristic $q$ is due to the fact that many derivatives vanish. More precisely, let $h \in\{0, \ldots, q-1\}$ s.t. $j=h$ mod. $q$. Then 


$$
\frac{d}{d^{i}} s^{j}= \begin{cases}\prod_{k=0}^{i-1}(j-k) s^{j-i} & \text { if } h \geq i \\ 0 & \text { if } h<i\end{cases}
$$

This was one of the reasons that motivated Hasse to introduce the following concept.

Definition 3. The $i$-th Hasse derivative of a polynomial $u(s)=\sum_{j=0}^{d} u_{j} s^{j}$ is defined as:

$$
\frac{\partial}{\partial^{i}} u(s)=\sum_{j=i}^{d}\left(\begin{array}{l}
j \\
i
\end{array}\right) u_{i} s^{j-i} .
$$

Observe that in characteristic 0 one has

$$
\frac{\partial}{\partial^{i}}=\frac{1}{i !} \frac{d}{d^{i}}
$$

Moreover, none of the Hasse derivatives vanishes identically for all polynomials, regardless of the characteristic of the base field, whereas in characteristic $q>0$, the $i$-th derivative of any polynomial is identically zero for all $i \geq q$.

It is therefore natural that we define the osculating normal curve in positive characteristic using the Hasse derivative instead of the normal derivative.

Definition 4. Let $\mathbb{F}$ be an algebraically closed field of characteristic $q>0$. The $o s$ culating normal curve $C_{p, m}$ is the closure of the image of the morphism

$$
\begin{aligned}
& \mathbb{F} \longrightarrow \operatorname{Grass}\left(p, \mathbb{F}^{m+p}\right) \\
& s \longmapsto \operatorname{rowsp}\left[\frac{\partial}{\partial i} s^{j}\right]_{i=0, \ldots, p-1 ; j=0, \ldots, m+p-1}
\end{aligned}
$$

where $\partial$ denotes the Hasse derivative.

For $p \leq 2$ the definition agrees with the one given at the beginning of this section. In particular, for $p=1$ we have a non-degenerate rational normal curve of degree $m$ in $\operatorname{Grass}\left(1, \mathbb{F}^{m+1}\right) \cong \mathbb{P}^{m}$. Notice also that the curve is well defined even if $p>q$, as we do not generate a zero row in the defining matrix.

Unfortunately, even with this adapted definition the osculating normal curve is degenerate for many choices of the parameters, as the following result points out:

Proposition 1. Let $\mathbb{F}$ be an algebraically closed field of characteristic $q>0$. Assume that $q \leq m$. Then the osculating normal curve $C_{p, m}$ is degenerate.

Proof. By Remark 2, it suffices to show that one of the minors of the matrix:

$$
\left[\left(\begin{array}{l}
j \\
i
\end{array}\right) s^{j-i}\right]_{i=0, \ldots, p-1 ; j=0, \ldots, m+p-1}
$$

is zero. Consider the sub-matrix consisting of columns $0, \ldots, p-2, c$, where $c$ is a multiple of $q, c \in\{p+1, \ldots, p+m\}$. The corresponding minor is:

$$
\operatorname{det}\left[\left(\begin{array}{l}
j \\
i
\end{array}\right)\right]_{i=0, \ldots, p-1 ; j=0, \ldots, p-2, c} s=\left(\begin{array}{c}
c \\
p-1
\end{array}\right) s=0 .
$$


The first equality follows from the observation that the matrix is upper triangular with ones on the diagonal, except for the entry in the lower right corner which equals $\left(\begin{array}{c}c \\ p-1\end{array}\right)$.

Remark 3. If $q \mid p$ and $m \geq p$, the minor of the sub-matrix consisting of columns $p-1, p+1, \ldots, 2 p-1$ equals

$$
\operatorname{det}\left[\left(\begin{array}{l}
j \\
i
\end{array}\right)\right]_{i=0, \ldots, p-1 ; j=p-1, p+1, \ldots, 2 p-1} s^{p^{2}-1}=p s^{p^{2}-1}=0 .
$$

The first equality follows from Lemma 9 in [3].

Remark 4. Degeneracy of the osculating normal curve over the field $\mathbb{F}_{q}$ with $q \leq$ $\max \{p, m\}$ also follows from Theorem 4

In Proposition 1 we saw that the osculating normal curve may be degenerate over a field $\mathbb{F}$ of positive characteristic $q$. Notice however that the curve may be non-degenerate for certain choices of the parameters $p, m$. The following example shows, e.g., that if the field $\mathbb{F}$ has characteristic $2, m=1$ and $p$ is odd, then $C_{p, 1}$ is non-degenerate.

Example 1. The curve $C_{p, 1}$ is the closure of the image of the morphism

$$
\begin{aligned}
\mathbb{F} & \longrightarrow \operatorname{Grass}\left(p, \mathbb{F}^{p+1}\right) \\
s & \longmapsto \operatorname{rowsp}\left[\left(\begin{array}{l}
j \\
i
\end{array}\right) s^{j-i}\right]_{i=0, \ldots, p-1 ; j=0, \ldots, p .}
\end{aligned}
$$

The minors of the matrix that defines the morphism are

$$
\left(\begin{array}{c}
p \\
i
\end{array}\right) s^{i} \text { for } i=0, \ldots, p
$$

Hence the curve is non-degenerate if and only if all the minors are non-zero, if and only if

$$
q \nmid\left(\begin{array}{c}
p \\
i
\end{array}\right) \text { for any } i=0, \ldots, p \text {. }
$$

Over a field of even characteristic, this is in fact the only case when the osculating normal curve is non-degenerate.

Corollary 1. Let $\mathbb{F}$ be an algebraically closed field of characteristic 2. Then the osculating normal curve $C_{p, m}$ is degenerate, unless $m=1$ and $p$ is odd. In the latter case, $C_{p, 1}$ is isomorphic to the rational normal curve of degree $p$ in $\mathbb{P}^{p}$. 


\subsection{Monomial systems and MDS matrices}

Definition 5. A matrix $M(s)=[N(s) D(s)]$ is monomial if the minors of all sizes of $M(s)$ are monomials. A system $G(s)$ associated to a monomial matrix $M(s)$ is called a monomial system.

A monomial matrix $M(s)=\left[\alpha_{i, j} s^{d_{i, j}}\right]$ is determined by:

- the coefficient matrix $M=\left[\alpha_{i, j}\right]$,

- the degree matrix $\left[d_{i, j}\right]$.

The degree matrix has the property that $d_{i, j}+d_{k, l}=d_{i, l}+d_{k, j}$ for all $i, j, k, l$.

Example 2. The osculating normal curve defines a monomial system.

Example 3. Let $\mathbb{F}$ be a field which contains at least three distinct elements $0,1, \alpha$. The matrix

$$
M(s)=\left[\begin{array}{cccc}
1 & 0 & s^{2} & \alpha s^{3} \\
0 & 1 & s & s^{2}
\end{array}\right]
$$

has minors

$$
1, s, s^{2},-s^{2}, \alpha s^{3},(1-\alpha) s^{4} .
$$

It therefore follows that $M(s)$ is a monomial matrix. A direct calculation shows that this system is non-degenerate.

Definition 6. A matrix $M$ with entries in $\mathbb{F}$ is Maximum Distance Separable (MDS) if all its maximal minors are non-zero.

Remark 5. In coding theory a linear code $C \subset \mathbb{F}^{n}$ is called an MDS code if all the maximal minors of the generator matrix of $C$ are non-zero. This explains the choice of the name for these matrices.

Remark 6. Let $M(s)$ be a monomial matrix. If the system associated to $M(s)$ is nondegenerate, then $M$ is an MDS matrix. This follows from Remark 2 It is not always the case that a monomial matrix $M(s)$ with MDS coefficient matrix $M$ is nondegenerate.

An example of degenerate $M(s)$ with MDS coefficient matrix is given in the following example.

Example 4. Let $\mathbb{F}=\mathbb{F}_{5}$, the finite field of 5 elements. The following monomial system defined by the matrix

$$
M(s)=\left[\begin{array}{llll}
1 & s & s & s^{2} \\
0 & 1 & 2 & 3 s
\end{array}\right]
$$

is left prime and has an MDS matrix as coefficient matrix. Nonetheless the system is degenerate as, e.g.,

$$
\left[\begin{array}{ll}
K_{1} & K_{2}
\end{array}\right]:=\left[\begin{array}{llll}
0 & 1 & 2 & 0 \\
0 & 0 & 0 & 1
\end{array}\right]
$$

results in the zero characteristic polynomial. 
In the next theorem we show that an MDS matrix of given size defined over a field $\mathbb{F}$ exists only if the ground field $\mathbb{F}$ has enough elements.

Theorem 4. Let $p, m \geq 2$ and let $M(s)$ be a monomial matrix of size $p \times(m+p)$ defined over a field $\mathbb{F}$ with q elements. If $q \leq \max \{p, m\}$, then $M(s)$ is degenerate.

Proof. If $M(s)$ is non-degenerate, then its coefficient matrix $M$ is MDS. Let $M^{\perp}$ be an $m \times(p+m)$-matrix defined over $\mathbb{F}$, such that $\operatorname{rowsp}(M)=\operatorname{ker}\left(M^{\perp}\right)$. Let $C^{\perp}$ be the dual code of $C$. The generator matrix of $C^{\perp}$ is then $M^{\perp}$. It is well known that $C$ is MDS if and only if $C^{\perp}$ is MDS. Therefore, $M^{\perp}$ is an MDS matrix.

We want to show that if $M$ (resp. $M^{\perp}$ ) is MDS of size $p \times(m+p)$ (resp. $m \times$ $(m+p))$ defined over $\mathbb{F}_{q}$, then $q \geq \max \{p+1, m+1\}$. The statement is symmetric in $p, m$, hence we can assume without loss of generality that $2 \leq p \leq m$. It suffices to prove that $q \geq m+1$.

We first consider the case $p=2$. Since $M$ is MDS, every pair of columns must be linearly independent. Over a field of $q$ elements, there are $q^{2}-1$ choices for the first column, $q^{2}-q$ for the second, $q^{2}-2(q-1)-1$ choices for the third, and so forth. Since there are $q^{2}-(m+1)(q-1)-1$ choices for the $m+2$-nd column, it must be $q^{2}-(m+1)(q-1)-1=(q-1)(q-m) \geq 1$, hence $q \geq m+1$.

For an arbitrary $p$, we can assume that the matrix $M$ is of the form $\left[I_{p} A\right]$, where $I_{p}$ is the $p \times p$ identity matrix and $A$ is a matrix of size $p \times m$. The MDS property of $M$ translates into the property that all the minors of all sizes of $A$ are non-zero. Consider the submatrix $N$ obtained from $M$ by deleting the last $p-2$ rows and the columns $3, \ldots, p, N=\left[\begin{array}{ll}I_{2} & B\end{array}\right]$ where $B$ consists of the first two rows of $A . N$ is a $2 \times(m+2)$ MDS matrix, since all the minors of all sizes of $B$ are non-zero. It follows that $q \geq m+1$ for every $p \geq 1$.

From the proposition it follows, e.g., that every monomial $M(s)$ of size $2 \times(m+$ 2 ) defined over $\mathbb{F}_{2}$ is degenerate, unless $m=1$. Clearly, there may be non-degenerate matrices which are not monomial. E.g., the following is an example of a $2 \times 4$ system defined over $\mathbb{F}_{2}$ which is non-degenerate:

Example 5. Consider the system defined over $\mathbb{F}_{2}$ by the matrix

$$
M(s)=\left[\begin{array}{llrr}
0 & s & s+1 & s^{2} \\
1 & s^{2}+1 & 1 & s
\end{array}\right] .
$$

The minors, listed in lexicographic order, are

$$
s, s+1, s^{2},\left(s^{3}+s+1\right), s^{4}, s .
$$

A direct computation shows that the system is non-degenerate.

We conclude the paper with the main result.

Theorem 5. Let $M(s)=[N(s) D(s)]$ be a monomial system having an MDS coefficient matrix $M$ of the form $M=\left[I_{p} R\right]$. Let the degrees of the coefficient matrix be $d_{i, j}=j-i$ if $j \geq i$ and zero else. Then $M(s)$ is non-degenerate of degree $m p$. 
Proof. Denote by $\alpha$ a multi-index $\alpha=\left(\alpha_{1}, \ldots, \alpha_{p}\right)$ with the property that

$$
1 \leq \alpha_{1}<\cdots \cdots \alpha_{p} \leq m+p .
$$

Denote by $m_{1}(s), \ldots, m_{p}(s)$ the $p$ row vectors of $M(s)$ and denote by $e_{1}, \ldots, e_{m+p}$ the canonical basis of $\mathbb{F}^{m+p}$. One readily verifies that the Plücker expansion of $M(s)$ has the form:

$$
m_{1}(s) \wedge \ldots \wedge m_{p}(s)=\sum_{\alpha \in\left\{\begin{array}{l}
n \\
p
\end{array}\right\}} m_{\alpha} e_{\alpha_{1}} \wedge \ldots \wedge e_{\alpha_{p}} s|\alpha|
$$

where $|\alpha|:=\sum_{i=1}^{p}\left(\alpha_{i}-i\right)$ and $m_{\alpha}$ is the minor of $M$ corresponding to the columns $\alpha_{1}, \ldots, \alpha_{p}$.

The multi-indices $\alpha$ have a natural partial order, coming from componentwise comparison of their entries. If $\beta=\left(\beta_{1}, \ldots, \beta_{p}\right)$ is a multi-index, then one defines:

$$
\alpha \leq \beta: \Longleftrightarrow \alpha_{i} \leq \beta_{i} \text { for } i=1, \ldots, p .
$$

By contradiction assume now that $M(s)$ is degenerate. Let $\left[K_{1} K_{2}\right]$ be a compensator which leads to the closed loop characteristic polynomial zero:

$$
\operatorname{det}\left[\begin{array}{cc}
K_{1} & K_{2} \\
N(s) & D(s)
\end{array}\right]=\sum_{\alpha} k_{\alpha} g_{\alpha}(s)=0 .
$$

In the last expansion $k_{\alpha}$ denotes up to sign the $m \times m$ minor of $\left[K_{1} K_{2}\right]$ corresponding to the columns $1 \leq \hat{\alpha}_{1}<\ldots<\hat{\alpha}_{m} \leq(m+p), \hat{\alpha}_{i} \notin\left\{\alpha_{1}, \ldots, \alpha_{p}\right\}$.

$\left[\begin{array}{ll}K_{1} & K_{2}\end{array}\right]$ has a well defined row reduced echelon form with Pivot indices $\hat{\beta}=$ $\left(\hat{\beta_{1}}, \ldots, \hat{\beta_{m}}\right)$. It follows that $k_{\alpha}=0$ for $\alpha \not \subset \beta$. But this means that the term $m_{\beta} s^{|\beta|}$ cannot cancel in the expansion (18) and this is a contradiction. $M(s)$ is therefore non-degenerate.

Remark 7. If $n>m p$ choose $d_{i, m+p}=n-m p+m+p-i$ in order to obtain once more a non-degenerate system of degree $n$.

By establishing the existence of a non-degenerate system, we have shown that Theorem 1 holds true for any algebraically closed field for $n=m p$.

Remark 8. In order to prove Theorem 1 in the situation when $n<m p$, one can show that for a generic system $(A, B, C)$ the set of dependent compensators, i.e., the set of compensators which results in a zero closed loop characteristic polynomial, has minimum possible dimension, namely $m p-n-1$. This is clearly sufficient to establish the result. In order to prove this statement, one can proceed in two ways. Either one shows that the condition is algebraic and constructs an example of a system of degree $n$ satisfying the condition. Alternativeley one shows that the coincidence set $S$ introduced in (12) has dimension $n^{2}+n(m+p)+m p-n-1$. The generic fiber of the projection onto the second factor has then dimension $m p-n-1$. This last argument was developed for the dynamic pole placement problem in [8]. 


\subsection{Non-degenerate systems over finite fields}

In this last subsection, we show that in general non-degeneracy does not guarantee that the pole placement map is surjective over a finite field.

Theorem 6. Let $\mathbb{F}_{2}$ be the binary field. Then no non-degenerate system defined over $\mathbb{F}_{2}$ induces an onto pole placement map:

$$
\operatorname{Grass}\left(2, \mathbb{F}_{2}^{4}\right) \longrightarrow \mathbb{P}^{4}\left(\mathbb{F}_{2}\right) .
$$

Proof. Let $M(s)$ be a non-degenerate matrix with entries in $\mathbb{F}_{2}[s]$. Let $\mathbb{F}$ denote the algebraic closure of $\mathbb{F}_{2}$ and let

$$
\chi: \operatorname{Grass}\left(2, \mathbb{F}^{4}\right) \longrightarrow \mathbb{P}^{4}(\mathbb{F})
$$

be the pole placement map associated to $M(s)$ over $\mathbb{F} \cdot \chi$ is a morphism, since $M(s)$ is non-degenerate. We will now show that the restriction of $\chi$ to $\mathbb{F}_{2}$-rational points

$$
\operatorname{Grass}\left(2, \mathbb{F}_{2}^{4}\right) \longrightarrow \mathbb{P}^{4}\left(\mathbb{F}_{2}\right)
$$

is never surjective.

Let $\operatorname{rowsp}(A) \in \operatorname{Grass}\left(2, \mathbb{F}_{2}^{4}\right)$. Denote by $A_{i, j}$ the determinant of the sub-matrix of $A$ consisting of columns $i$ and $j$. Then:

$$
\chi(A)=\left[\sum_{i<j} \chi_{i j k} A_{i, j}\right]_{k=0, \ldots, 4}
$$

where

$$
\operatorname{det}\left[\begin{array}{c}
M(s) \\
A
\end{array}\right]=\sum_{k=0}^{4} \sum_{i<j} \chi_{i j k} A_{i, j} s^{k} .
$$

Since the system is non-degenerate, the $5 \times 6$ matrix:

$$
C=\left[\begin{array}{ccc}
\chi_{120} & \ldots & \chi_{340} \\
\vdots & & \vdots \\
\chi_{124} & \ldots & \chi_{344}
\end{array}\right]
$$

has full rank, hence its kernel is 1-dimensional and generated by a unique element of $\mathbb{F}_{2}^{6}$. By non-degeneracy, the generator of the kernel corresponds to a point in $\mathbb{P}^{6}\left(\mathbb{F}_{2}\right)$ which does not belong to $\operatorname{Grass}\left(2, \mathbb{F}^{4}\right)$. Hence we have the following possibilities for the generator of $\operatorname{ker} C$ :

$$
\begin{aligned}
& (1,0,0,0,0,1),(0,1,0,0,1,0),(0,0,1,1,0,0),(1,1,1,1,1,1), \\
& (1,1,0,0,0,1),(1,0,1,0,0,1),(1,0,0,1,0,1),(1,0,0,0,1,1), \\
& (1,1,0,0,1,0),(0,1,1,0,1,0),(0,1,0,1,1,0),(0,1,0,0,1,1),
\end{aligned}
$$




$$
\begin{aligned}
& (1,0,1,1,0,0),(0,1,1,1,0,0),(0,0,1,1,1,0),(0,0,1,1,0,1), \\
& (0,0,1,1,1,1),(0,1,0,1,1,1),(0,1,1,0,1,1),(0,1,1,1,0,1), \\
& (1,0,0,1,1,1),(1,0,1,0,1,1),(1,0,1,1,1,0),(1,1,0,1,0,1), \\
& (1,1,0,1,1,0),(1,1,1,0,0,1),(1,1,1,0,1,0),(1,1,1,1,0,0) .
\end{aligned}
$$

Observe that the problem is symmetric with respect to the following changes of basis of $\mathbb{F}_{2}^{6}=\left\langle e_{12}, \ldots, e_{34}\right\rangle$ (which correspond to automorphisms of $\operatorname{Grass}\left(2, \mathbb{F}^{4}\right)$ ) and composition thereof:

- exchange $e_{12}$ and $e_{34}$ and leave the rest unaltered,

- exchange $e_{13}$ and $e_{24}$ and leave the rest unaltered,

- exchange $e_{14}$ and $e_{23}$ and leave the rest unaltered,

- exchange $e_{12}$ and $e_{13}$, exchange $e_{34}$ and $e_{24}$,

- exchange $e_{12}$ and $e_{14}$, exchange $e_{34}$ and $e_{23}$,

- exchange $e_{13}$ and $e_{14}$, exchange $e_{24}$ and $e_{23}$.

Hence, reducing to the analysis of the following possibilities is non-restrictive:

$$
(1,0,0,0,0,1),(1,1,0,0,0,1),(0,0,1,1,1,1),(1,1,1,1,1,1) .
$$

Up to a change of coordinates in $\mathbb{P}^{4}$, we may assume that the corresponding matrix $C$ is respectively:

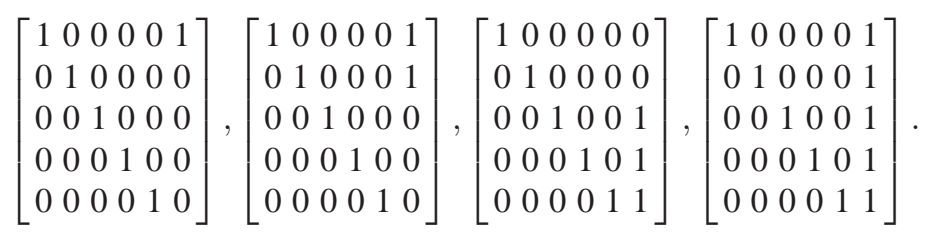

Analyzing each case, it is now easy to prove that the corresponding $\chi$ is not onto. E.g., in the first case we have:

$$
\chi(A)=\left[A_{1,2}+A_{3,4}, A_{1,3}, A_{1,4}, A_{2,3}, A_{2,4}\right]
$$

which is surjective if and only if the equations

$$
\begin{gathered}
A_{1,2}+A_{3,4}=\alpha_{0}, A_{1,3}=\alpha_{1}, A_{1,4}=\alpha_{2}, A_{2,3}=\alpha_{3}, A_{2,4}=\alpha_{4}, \\
A_{1,2} A_{3,4}+A_{1,3} A_{2,4}+A_{1,4} A_{2,3}=0
\end{gathered}
$$

have a solution in $\mathbb{F}_{2}^{6}$ for any choice of $\left[\alpha_{0}: \ldots: \alpha_{4}\right] \in \mathbb{P}^{4}\left(\mathbb{F}_{2}\right)$. Letting $x=A_{1,2}$, the equations reduce to:

$$
x^{2}+\alpha_{0} x+\alpha_{1} \alpha_{4}+\alpha_{2} \alpha_{3}=0
$$

which has no solution over $\mathbb{F}_{2}$ for $\alpha_{0}=\alpha_{1}=\alpha_{2}=\alpha_{4}=1, \alpha_{3}=0$.

In the second case we have:

$$
\chi(A)=\left[A_{1,2}+A_{3,4}, A_{1,3}+A_{3,4}, A_{1,4}, A_{2,3}, A_{2,4}\right]
$$


which is surjective if and only if the equations

$$
\begin{gathered}
A_{1,2}+A_{3,4}=\alpha_{0}, A_{1,3}+A_{3,4}=\alpha_{1}, A_{1,4}=\alpha_{2}, A_{2,3}=\alpha_{3}, A_{2,4}=\alpha_{4}, \\
A_{1,2} A_{3,4}+A_{1,3} A_{2,4}+A_{1,4} A_{2,3}=0
\end{gathered}
$$

have a solution in $\mathbb{F}_{2}^{6}$ for any choice of $\left[\alpha_{0}: \ldots: \alpha_{4}\right] \in \mathbb{P}^{4}\left(\mathbb{F}_{2}\right)$. Letting $x=A_{3,4}$, the equations reduce to:

$$
x^{2}+\left(\alpha_{0}+\alpha_{4}\right) x+\alpha_{1} \alpha_{4}+\alpha_{2} \alpha_{3}=0,
$$

which has no solution over $\mathbb{F}_{2}$ for $\alpha_{0}=\alpha_{1}=\alpha_{2}=\alpha_{3}=1, \alpha_{4}=0$.

In the third case we have:

$$
\chi(A)=\left[A_{1,2}, A_{1,3}, A_{1,4}+A_{3,4}, A_{2,3}+A_{3,4}, A_{2,4}+A_{3,4}\right]
$$

which is surjective if and only if the equations

$$
\begin{gathered}
A_{1,2}=\alpha_{0}, A_{1,3}=\alpha_{1}, A_{1,4}+A_{3,4}=\alpha_{2}, A_{2,3}+A_{3,4}=\alpha_{3}, A_{2,4}+A_{3,4}=\alpha_{4}, \\
A_{1,2} A_{3,4}+A_{1,3} A_{2,4}+A_{1,4} A_{2,3}=0
\end{gathered}
$$

have a solution in $\mathbb{F}_{2}^{6}$ for any choice of $\left[\alpha_{0}: \ldots: \alpha_{4}\right] \in \mathbb{P}^{4}\left(\mathbb{F}_{2}\right)$. Letting $x=A_{3,4}$, the equations reduce to:

$$
x^{2}+\left(\alpha_{0}+\alpha_{1}+\alpha_{2}+\alpha_{3}\right) x+\alpha_{1} \alpha_{4}+\alpha_{2} \alpha_{3}=0,
$$

which has no solution over $\mathbb{F}_{2}$ for $\alpha_{0}=\alpha_{2}=\alpha_{3}=0, \alpha_{1}=\alpha_{4}=1$.

In the last case we have:

$$
\chi(A)=\left[A_{1,2}+A_{3,4}, A_{1,3}+A_{3,4}, A_{1,4}+A_{3,4}, A_{2,3}+A_{3,4}, A_{2,4}+A_{3,4}\right]
$$

which is surjective if and only if the equations

$$
\begin{gathered}
A_{1,2}+A_{3,4}=\alpha_{0}, A_{1,3}+A_{3,4}=\alpha_{1}, A_{1,4}+A_{3,4}=\alpha_{2}, A_{2,3}+A_{3,4}=\alpha_{3}, A_{2,4}+A_{3,4}=\alpha_{4}, \\
A_{1,2} A_{3,4}+A_{1,3} A_{2,4}+A_{1,4} A_{2,3}=0
\end{gathered}
$$

have a solution in $\mathbb{F}_{2}^{6}$ for any choice of $\left[\alpha_{0}: \ldots: \alpha_{4}\right] \in \mathbb{P}^{4}\left(\mathbb{F}_{2}\right)$. Letting $x=A_{3,4}$, the equations reduce to:

$$
x^{2}+\left(\alpha_{0}+\alpha_{1}+\alpha_{2}+\alpha_{3}+\alpha_{4}\right) x+\alpha_{1} \alpha_{4}+\alpha_{2} \alpha_{3}=0,
$$

which has no solution over $\mathbb{F}_{2}$ for $\alpha_{0}=\alpha_{1}=\alpha_{4}=1, \alpha_{2}=\alpha_{3}=0$.

Acknowledgements The work of both authors is supported by the Swiss National Science Foundation through grants \#123393, \#113251, and \#107887. 
Pole Placement with Fields of Positive Characteristic

\section{References}

1. Brockett, R.W., Byrnes, C.I.: Multivariable Nyquist criteria, root loci and pole placement: A geometric viewpoint. IEEE Trans. Automat. Control AC-26, 271-284 (1981)

2. Byrnes, C.I.: Pole assignment by output feedback. In: H. Nijmeijer, J.M. Schumacher (eds.) Three Decades of Mathematical System Theory, Lecture Notes in Control and Information Sciences \# 135, pp. 31-78. Springer Verlag (1989)

3. Gessel, I., Viennot, G.: Binomial determinants, paths, and hook length formulae. Adv. in Math. 58(3), 300-321 (1985)

4. Kleiman, S.L.: Problem 15: Rigorous foundations of Schubert's enumerative calculus. In: Proceedings of Symposia in Pure Mathematics, vol. 28, pp. 445-482. Am. Math. Soc. (1976)

5. Martin, C.F., Hermann, R.: Applications of algebraic geometry to system theory: The McMillan degree and Kronecker indices as topological and holomorphic invariants. SIAM J. Control Optim. 16, 743-755 (1978)

6. Mumford, D.: Algebraic Geometry I: Complex Projective Varieties. Springer Verlag, Berlin, New York (1976)

7. Rosenthal, J.: Geometric methods for feedback stabilization of multivariable linear systems. Ph.D. thesis, Arizona State University (1990)

8. Rosenthal, J.: On dynamic feedback compensation and compactification of systems. SIAM J. Control Optim. 32(1), 279-296 (1994)

9. Rosenthal, J., Schumacher, J.M.: Realization by inspection. IEEE Trans. Automat. Contr. AC-42(9), 1257-1263 (1997)

10. Rosenthal, J., Sottile, F.: Some remarks on real and complex output feedback. Systems \& Control Letters 33(2), 73-80 (1998)

11. Schubert, H.: Anzahlbestimmung für lineare Räume beliebiger Dimension. Acta Math. 8, 97-118 (1886)

12. Schubert, H.: Beziehungen zwischen den linearen Räumen auferlegbaren charakteristischen Bedingungen. Math. Ann. 38, 598-602 (1891)

13. Shafarevich, I.R.: Basic algebraic geometry. 1, second edn. Springer-Verlag, Berlin (1994). Varieties in projective space, Translated from the 1988 Russian edition and with notes by Miles Reid

14. Wang, X.: Pole placement by static output feedback. Journal of Math. Systems, Estimation, and Control 2(2), 205-218 (1992)

15. Wang, X.: Grassmannian, central projection and output feedback pole assignment of linear systems. IEEE Trans. Automat. Contr. AC-41(6), 786-794 (1996) 